Fernanda Lima

Modelagem Semântica de Aplicações na WWW

Tese de Doutorado

Tese apresentada como requisito parcial para obtenção do título de Doutor pelo Programa de Pós-Graduação em Informática da PUC-Rio.

Orientador: Daniel Schwabe 
Fernanda Lima

Modelagem Semântica de Aplicações na WWW

Tese apresentada como requisito parcial para obtenção do título de Doutor pelo Programa de Pós-Graduação em Informática da PUC-Rio. Aprovada pela Comissão Examinadora abaixo assinada.

Daniel Schwabe

Orientador

PUC-Rio

Alberto H. F. Laender UFMG

Marco Antonio Casanova

PUC-Rio

Marta L. Queirós Mattoso

COPPE/UFRJ

Simone Diniz Junqueira Barbosa

PUC-Rio

Prof. Ney Dumont

Coordenador Setorial do Centro Técnico Científico - PUC-Rio

Rio de Janeiro, 28 de março de 2003 
Todos os direitos reservados. É proibida a reprodução total ou parcial do trabalho sem autorização do autor, do orientador e da universidade.

\section{Fernanda Lima}

Cursou Tecnólogo em Processamento de Dados na PUC-Rio em 1983. Atuou em empresas como Analista de Sistemas, Administradora de Bancos de Dados, Analista de Suporte, Programadora de 1984 a 1997. Retornou ao mundo acadêmico para realizar Mestrado em Engenharia de Sistemas e Computação na COPPE/Sistemas, UFRJ, encerrado em 1996 com publicações na área de Banco de Dados. Ministrou disciplinas relacionadas à Web em diversos cursos, inclusive no Bacharelado em Informática da PUCRio. Coordenou curso de Desenvolvimento de Aplicações na Web na CCE PUC-Rio. Possui interesse acadêmico e profissional nas áreas de Engenharia de Software, Orientação a Objetos e Web Semântica.

Ficha Catalográfica

Lima, Fernanda

Modelagem semântica de aplicações na WWW / Fernanda Lima; orientador: Daniel Schwabe. Rio de Janeiro : PUC, Departamento de Informática, 2003.

128 f. : il. ; $30 \mathrm{~cm}$

Tese (doutorado) - Pontifícia Universidade Católica do Rio de Janeiro, Departamento de Informática.

Inclui referências bibliográficas.

1. Informática - Teses. 2. Web semântica. 3 . Projeto de aplicações web. 4. SHDM. 5. OOHDM. 6. Web. 7. World Wide Web. I. Schwabe, Daniel. II. Pontifícia Universidade Católica do Rio de Janeiro. Departamento de Informática. III. Título. 
Este trabalho é dedicado a meu marido Ortwin e meu filho Kristian, por existirem e tornarem o mundo mais belo a cada dia. A vocês, com todo amor que houver nesta vida! 


\section{Agradecimentos}

Ao meu orientador, Professor Daniel Schwabe, pelo incentivo e parceria para a realização deste trabalho, e também pelo ombro amigo em momentos difíceis.

Ao CNPq, à Capes e à PUC-Rio, pelos auxílios concedidos, sem os quais este trabalho não poderia ter sido realizado.

Aos professores que participaram da Comissão examinadora. Em especial, aos meus ex-orientadores Marta Mattoso, Casanova e Daniel Schwabe, por terem servido de exemplo e inspiração para minha formação acadêmica.

A todos os professores, funcionários e alunos do Departamento de Informática pela convivência agradável de todos os dias.

Ao meus amigos e colegas do laboratório Webeng/TecWeb: Natacha, Patrícia Vilain, Mark, Condack e Guilherme pelo companheirismo de todas as horas e em especial à Adriana, pelo carinho, presença e ativa colaboração na etapa final. A todos os meus amigos e colegas da PUC-Rio. Aos meus amigos da Dataprev, que me acompanharam após a saída da empresa, em especial, à Ana Caldas, Cristina, Gabriela, Lia, Patrícia e Renato. A minha querida amiga Simone, companheira de muitas lutas e estradas semelhantes. A todos os meus outros amigos e colegas, não mencionados nominalmente aqui, mas que sempre me trataram com carinho e foram correspondidos.

As minhas queridas mãe Pilar e irmã Vera por serem exemplos de luta e fortaleza.

A meus queridos pai Hemar e irmão André pela saudade e pela presença em meu coração.

A meu amado marido Ortwin, que não só me acompanhou durante esta jornada, mas também foi fonte de perseverança, inspiração e muito amor.

A meu pequeno filho Kristian, que trouxe uma luz linda em minha vida e os momentos mais felizes que já vivi. 


\section{Resumo}

Lima, Fernanda. Modelagem Semântica de Aplicações na WWW. Rio de Janeiro, 2003. 128p. Tese de Doutorado - Departamento de Informática, Pontifícia Universidade Católica do Rio de Janeiro.

Este trabalho apresenta um modelo para projeto e implementação de aplicações hipermídia no contexto da Web semântica. A partir dos princípios do "Object Oriented Hypermedia Design Method", utilizamos as noções de ontologias para definir o modelo conceitual de uma aplicação, estendendo o poder expressivo daquele método. Os modelos de navegação são definidos utilizando-se uma linguagem de consulta que permite referências tanto ao esquema de dados quanto às suas instâncias, possibilitando a definição de estruturas de navegação flexíveis e abrangentes. Adicionalmente, propomos a utilização de estruturas de acesso facetadas para o apoio à escolha de objetos de navegação utilizando múltiplos critérios. Finalmente, apresentamos uma arquitetura de implementação que permite a utilização direta da especificação da aplicação na derivação da implementação da aplicação final.

\section{Palavras-chave}

Web Semântica, Projeto de Aplicações Web, SHDM, OOHDM, Web, WWW. 


\section{Abstract}

Lima, Fernanda. Modelagem Semântica de Aplicações na WWW. Rio de Janeiro, 2003. 128p. Tese de Doutorado - Departamento de Informática, Pontifícia Universidade Católica do Rio de Janeiro.

In this thesis we present a method for the design and implementation of web applications for the Semantic Web. Based on the "Object Oriented Hypermedia Design Method" approach, we used ontology concepts to define an application conceptual model, extending the expressive power of the original method. The navigational models definitions use a query language capable of querying both schema and instances, enabling the specification of flexible access structures. Additionally, we propose the use of faceted access structures to improve the selection of navigational objects organized by multiple criteria. Finally, we present an implementation architecture that allows the direct use of the application specifications when deriving a final application implementation.

\section{Keywords}

Semantic Web, Web Application Design, SHDM, OOHDM, Web, WWW. 


\section{Sumário}

1 Introdução 15

1.1. A Web Atual 17

1.2. A Web Semântica 18

1.3. Contribuições 19

1.4. Estrutura da Tese 20

2 Fundamentos $\quad 22$

2.1. Ontologias 22

2.2. Linguagens para Especificação de Ontologias 23

2.2.1. XML 25

2.2.2. XML Schema 25

2.2.3. RDF e RDF(S) 26

2.2.4. DAML+OIL 30

2.2.5. Comparação 34

2.3. Linguagens de Consulta 38

2.3.1. RQL 39

2.3.2. Comparação com outras linguagens de consulta 40

2.4. Arquiteturas de Implementação 40

2.4.1. ICS-FORTH 40

2.4.2. Sesame 41

2.4.3. SeBOR 41

2.4.4. Comparação com outras Arquiteturas de Implementação para RDF(S) 42

2.5. Description Logics 42

2.6. Facetas 44

2.7. Projeto de Aplicações Hipermídia e Aplicações Web 45

2.7.1. Métodos para Projeto de Aplicações Hipermídia e Aplicações Web 46

2.7.2. O Método OOHDM 47

3 O Método SHDM 53

3.1. Projeto Conceitual SHDM resumido 54

3.2. Projeto Navegacional SHDM resumido 56 
3.3. Comentários Adicionais 59

3.4. Comparação entre OOHDM e SHDM 60

4 O Projeto Conceitual SHDM 62

4.1. Esquema Conceitual 62

4.1.1. Subclasse 63

4.1.2. Subrelacionamento 64

4.1.3. Multiplicidade de Atributo 66

4.1.4. Tipos de Dados utilizando XML Schema 67

4.1.5. Enumeração de Atributo 68

4.1.6. Classe Inferida 68

4.1.7. Estereótipo "ArbitraryClassHierarchy" 70

$\begin{array}{ll}\text { 4.1.8. Relacionamento Inverso } & 71\end{array}$

4.2. Exemplo resumido da Ontologia Conceitual 72

4.3. Instâncias 73

5 O Projeto Navegacional SHDM $\quad 74$

5.1. Esquema de Classes Navegacionais $\quad 77$

5.1.1. Classe Navegacional ou Nó $\quad 77$

5.1.2. Subrelacionamento Navegacional $\quad 78$

5.2. Esquema de Contextos Navegacionais 80

5.2.1. Estrutura de Acesso 81

5.2.2. Contexto Navegacional 82

5.2.3. Estrutura de Acesso Facetada 83

5.2.4. Cartão de Especificação de Estrutura de Acesso Facetada 85

5.2.5. Contexto Navegacional Facetado 88

5.2.6. Outros Cartões de Especificação (Contexto, Contexto Facetado e

Estrutura de Acesso) $\quad 89$

5.3. Comparação entre Esquema de Contextos Navegacionais

$\begin{array}{ll}\text { SHDM e OOHDM } & 90\end{array}$

6 Arquitetura de Implementação $\quad 99$

6.1. Visão Geral da Arquitetura de Implementação 99

6.2. Implementação Atual 102

$\begin{array}{ll}\text { 6.3. Experimento Realizado } & 104\end{array}$

7 Conclusões 106

7.1. Trabalhos Relacionados 106 
7.2. Contribuições

7.3. Trabalhos Futuros

Referências Bibliográficas

Apêndice

A.1 Ontologia de Artes

117

A.2 Ontologia de Vinhos

Anexos 


\section{Lista de Abreviaturas e Siglas}

\begin{tabular}{|l|l|}
\hline DAML & DAML DARPA Agent Markup Language \\
\hline DL & Description Logics \\
\hline DTD & Data Type Definition \\
\hline HTML & HyperText Markup Language \\
\hline OIL & Ontology Inference Layer \\
\hline OOHDM & Object Oriented Hypermedia Design Method \\
\hline OWL & Web Ontology Language \\
\hline RDF & Resource Description Framework \\
\hline RDF(S) & Resource Description Framework Schema \\
\hline RDQL & RDF Data Query Language \\
\hline RQL & RDF Query Language \\
\hline SHDM & Semantic Hypermedia Design Method \\
\hline URI & Universal Resource Identifier \\
\hline URL & Uniform Resource Locator \\
\hline W3C & World Wide Web Consortium \\
\hline Web & World Wide Web \\
\hline WWW & World Wide Web \\
\hline XML & eXtensible Markup Language \\
\hline XML Schema & eXtensible Markup Schema Language \\
\hline
\end{tabular}




\section{Lista de figuras}

Figura 1 - Exemplo RDF em representação gráfica de grafo .........................28

Figura 2 - Exemplo RDF em formato serializado RDF/XML .........................28

Figura 3 - Exemplo de um esquema RDF(S) [Gil \& Ratnakar, 2001] ..............29

Figura 4 - Exemplo de DAML+OIL [Gil \& Ratnakar, 2001] ...........................33

Figura 5 - Cartão de contexto ou grupo de contexto ......................................51

Figura 6 - Cartão de Estrutura de Acesso ......................................................51

Figura 7 - Cartão de Estrutura de Acesso hierárquica ..................................52

Figura 8. Modelos SHDM em camadas ....................................................53

Figura 9 - Notação de subclasse (como na UML) …...................................63

Figura 10 - Exemplo de subclasse .............................................................64

Figura 11 - Notação de Subrelacionamento................................................64

Figura 12 - Exemplo de Subrelacionamento ..............................................65

Figura 13 - Exemplo de Multiplicidade de Atributo .........................................66

Figura 14 - Exemplo de Tipos de Dados utilizando XML Schema...................67

Figura 15 - Exemplo de Enumeração de Atributo ........................................68

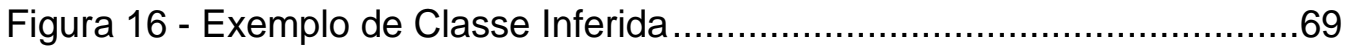

Figura 17 - Exemplo de hierarquia de profundidade arbitrária .......................71

Figura 18 - Notação de Relacionamento Inverso .........................................72

Figura 19 - Exemplo de Relacionamento Inverso .......................................72

Figura 20 - Trecho da Ontologia Conceitual de Artes .................................73

Figura 21 - Trecho das instâncias da Ontologia Conceitual de Artes .............73

Figura 22 - Exemplo de facetas .............................................................76

Figura 23 - Exemplo de Classe Navegacional ou Nó .....................................78

Figura 24 - Exemplo de Subrelacionamento Navegacional............................79

Figura 25 - Exemplo de Estrutura de Acesso...................................................81

Figura 26 - Exemplo de Contexto Navegacional .........................................82

Figura 27 - Exemplo de Estrutura de Acesso Facetada ..................................84

Figura 28 - Exemplo de Cartão de Especificação de Estrutura de Acesso Facetada. .86

Figura 29 - Exemplo RDF/XML de especificação da estrutura de uma faceta.

Figura 30 - Exemplo de Contexto Navegacional Facetado .89 
Figura 31 - Esquema de Contextos Navegacionais SHDM da Ontologia de Artes .90

Figura 32 - Esquema de Contextos Navegacionais OOHDM da Ontologia de Artes 91

Figura 33 - Esquema de Contextos Navegacionais SHDM . .92

Figura 34 - Esquema de Contextos Navegacionais OOHDM. .92

Figura 35 - Vocabulário de navegação do SHDM .97

Figura 36 - Meta esquema do SHDM .98

Figura 37 - Arquitetura de implementação para os artefatos SHDM 100

Figura 38 - Ambiente de armazenamento, inferência e consulta 101

Figura 39 - Arquitetura Sesame com OMM e BOR [Kiryakov et al., 2002a e 2002b]. 103

Figura 40 - Esboço de template para Contexto de Artefato Alfabético 104

Figura 41 - Instância do template para a implementação da ontologia de artes 105

Figura 42 - Esquema de Classes Conceituais da ontologia de artes 117

Figura 43 - Esquema de Classes Navegacionais da ontologia de Artes 118

Figura 44 - Esquema de Contextos da ontologia de artes 119

Figura 45 - Cartão de Estrutura de Acesso. 119

Figura 46 - Cartão de Contexto. 120

Figura 47 - Classe Conceitual Wine com enumeração de atributos 121

Figura 48 - Esquema de Classes Conceituais da ontologia de vinhos (preliminar)

Figura 49 - Esquema de Classes Conceituais da ontologia de vinhos (final) 122 Figura 50 - Esquema de Classes Navegacionais da ontologia de vinhos ....123 Figura 51 - Esquema de Contextos Navegacionais do domínio de vinhos...125 


\section{Lista de tabelas}

Tabela 1 - A Evolução da Web [TopQuadrant 2003] ...................................18

Tabela 2- Comparação resumida da funcionalidade das linguagens XML DTD, XML Schema, RDF(S), DAML+OIL e OWL ......................................34

Tabela 3 - Resumo da comparação entre as linguagens XML Schema, RDF(S) e DAML+OIL [Gil\&Ratnakar2002] .........................................38

Tabela 4 - Exemplos de Consultas RQL ..................................................... 40

Tabela 5 - Etapas SHDM e seus Artefatos …..........................................54

Tabela 6 - Resumo de Comparaçao entre UML x DAML+OIL ......................60

Tabela 7 - Exemplos de Consultas RQL para o domínio de vinhos...............124

Tabela 8 - Comparação entre Ferramentas/Ambientes para Armazenamento e Consulta de Ontologias [Magkanaraki et al 2002] ..............................127

Tabela 9-Comparação entre poder expressivo de linguagens de consulta para RDF(S) [Magkanaraki et al. 2002 .................................................128 